\title{
Gamificación y derecho procesal: ¿diversión o perversión?
}

\section{Gaming and procedural law: joy or perversion?}

\author{
Ana Isabel Blanco García ${ }^{1}$, Raquel Borges Blázquez², Elena de Luis García ${ }^{3}$ \& \\ Elisa Simó Soler ${ }^{4}$
}

Fecha de recepción: 04/03/2020; Fecha de revisión: 05/05/2020; Fecha de aceptación: 08/09/20

Cómo citar este artículo:

Blanco, A.I., Borges, R., De Luis, E. \& Simó, E. (2020). Gamificación y derecho procesal. Revista de Innovación y Buenas Prácticas Docentes, 9(2), 46-60.

Autor de Correspondencia: A.Isabel.Blanco@uv.es

\section{Resumen:}

El presente proyecto ha consistido en la incorporación, dentro del proceso de enseñanza-aprendizaje de la asignatura de Derecho Procesal, de un sistema de gamificación a través de la herramienta Kahoot. Su utilización ha servido para comprobar la utilidad de la aplicación en la docencia de una materia compleja, incluso en un contexto socio-educativo marcado por la crisis sanitaria consecuencia del Covid-19. Esta herramienta gratuita permite fomentar la competitividad entre los/las estudiantes e incentivar una mayor participación, con repercusiones positivas para la organización del estudio de los exámenes.

Asimismo, este proyecto ha permitido analizar si el idioma en que se imparte la docencia -castellano, valenciano e inglés- constituye un factor clave en los resultados obtenidos por el alumnado o su implicación en la asignatura.

Es importante destacar que la herramienta Kahoot permite la integración de los dispositivos móviles en su proceso de aprendizaje, dejando de lado sus usos menos apropiados para la docencia, de igual forma que fomenta la interacción, participación y compromiso con la asignatura, contribuyendo su retroalimentación a una mejora del rendimiento del alumnado.

Palabras clave: derecho procesal, docencia online, enseñanza-aprendizaje, gamificación.

\begin{abstract}
:
The present project consists in incorporating, within the teaching-learning process of the Procedural Law subject, a game-based learning platform through the Kahoot tool. The experience has allowed to verify the usefulness of the application in the teaching of a complex subject, even in a socio-educational context affected by the health crisis due to the Covid-19. This free tool enables the promotion of competitiveness among students and encourages greater participation, with positive impact on the organization of the study of exams.

Likewise, this project has analysed whether the language in which the teaching is given -Spanish, Valencian and English- is a key factor in the results obtained by the students or their interest in the subject.

It is important to highlight that the Kahoot tool allows the students to integrate mobile devices in their learning process, leaving aside their less appropriate uses for teaching, as well as promoting interaction, participation and commitment with the class, contributing the feedback to an improvement in the students' output.
\end{abstract}

Key Words: procedural law, online learning, teaching-learning, gamification.

\footnotetext{
${ }^{1}$ Universitat de València (España), A.Isabel.Blanco@uv.es; CÓDIGO ORCID: 0000-0002-8313-4930

2 Universitat de València (España), Raquel.Borges@uv.es; CÓDIGO ORCID: 0000-0003-4899-406X

3 Universitat de València (España), Elena.Deluis@uv.es; CÓDIGO ORCID: 0000-0001-7987-957X

4 Universitat de València (España), Elisa.Simo@uv.es; CÓDIGO ORCID: 0000-0001-5606-7085
} 


\section{INTRODUCCIÓN}

El objetivo principal de este proyecto de innovación docente es la introducción de un nuevo enfoque pedagógico, basado en el juego y la gamificación educativa (Lee et al., 2011) a través de la implantación de Kahoot como método de evaluación de cada una de las unidades didácticas que lo componen, a través de la realización de una serie de preguntas y respuestas proyectadas por el/la profesor/a -de forma breve y concisa- y resueltas por el alumnado a través de sus teléfonos móviles. Herramientas como Kahoot, disponible y accesible para el público en general y construida de forma muy intuitiva para que resulte no solo útil sino fácil de gestionar (Carreras, 2017). Todo ello con el fin de captar el interés de los/las alumnos/as por la asignatura, mejorar su constancia de estudio, motivación, atención y participación activa en las clases (Barros, 2016; Andreu, 2020).

La introducción de las TICs en la educación ha supuesto un cambio de paradigma en la forma de enseñar (Moya, 2018). Si hasta ahora no se prestaba tanta atención a la adquisición de competencias y habilidades -no solo académicas sino también sociales-, la utilización de las TICs en las aulas lo ha tornado imprescindible (Moya, 2016). La docencia universitaria ya no es simplemente un aprendizaje de las teorías y conceptos básicos de las materias, sino que busca educar y, más importante aún, formar al alumnado como persona y futuro trabajador/a, dotado/a de herramientas necesarias para una mayor competencia en el desempeño de sus actividades (Dalmases, 2017; Catalán, 2019).

Con la intención de continuar formándonos como docentes del siglo XXI se solicitó el proyecto docente que lleva por título "Gamificación de la docencia: Kahoot y Plickers aplicados al Derecho procesal en castellano, valenciano e inglés". Este proyecto nació con el ánimo de innovar en la gamificación de la docencia (Lee y Hammer, 2011, 59; Deterding et al, 2011; Bastante y Moreno, 2020) en los idiomas en que se imparte la asignatura de Derecho Procesal, utilizando Kahoot y Plickers, aunando la modernización de la sociedad y la incorporación de móviles con nuevas metodologías de enseñanza-aprendizaje, potenciando la adquisición de conocimientos y de habilidades para la profesión. Aunque el título del proyecto incluía Kahoot y Plickers, la declaración del estado de alarma el 14 de marzo de 2020 por la crisis del coronavirus nos forzó a la docencia online, imposibilitando el uso de la aplicación Plickers. Es por ello que, a partir de ahora, únicamente nos referiremos a la aplicación Kahoot, al ser ésta la única que hemos podido utilizar en el desarrollo de la experiencia docente.

Kahoot es una de las aplicaciones móviles que mejor valoración por parte del alumnado está recibiendo, una herramienta útil y divertida que utiliza una metodología empírica-analítica y que fomenta la motivación del alumnado para el estudio de la asignatura de Derecho Procesal (Calabrese, 2015). Esta herramienta metodológica innovadora no solo facilita la interacción entre el propio alumnado, sino también constituye un elemento motivacional (Sánchez, 2015) para la consecución de objetivos concretos, tales como la consolidación de los conocimientos adquiridos durante las clases magistrales, amén de una retroalimentación inmediata (Vérez, 2016).

\section{DESARROLLO DE LA EXPERIENCIA DE INNOVACIÓN}

La asignatura escogida para la realización de la experiencia docente es "Derecho Procesal I (Introducción)" del Grado en Derecho de la Facultat de Dret de la Universitat de València. La muestra incluye todas las lenguas en las que se imparte docencia en la Universidad de Valencia: inglés, valenciano y castellano. El objetivo, por tanto, es doble. 
Por una parte, abordar todos los perfiles de estudiantes que se matriculan en nuestra facultad. Por otra parte, testar si la lengua vehicular de la docencia tiene incidencia en la impartición de la asignatura de Derecho Procesal I. Ninguno de los grupos comparte docente, aun planteándose de igual forma la actividad. No obstante, por regla general, el grupo AR (inglés) suele tener mayor índice de asistencia y mejor rendimiento que el resto de grupos en castellano o valenciano. En este sentido, el número de estudiantes que participó en la experiencia coincide con el de asistentes al curso. Los porcentajes de participación son dispares. Así, en el grupo AR la participación fue del 92\%, mientras que en el de valenciano fue del $80 \%$ y del de castellano fue del $56,25 \%$. La elevada tasa de participación se ve favorecida porque son estudiantes con un alto grado de alfabetización tecnológica, lo que incrementa el potencial de esta herramienta tan intuitiva.

En el grupo en inglés contamos con 61 matriculados/as, de los cuales 10 abandonaron el curso a consecuencia de la crisis del Covid-19, al tratarse de estudiantes beneficiados/as por una beca Erasmus, para cuatro de los cuales el inglés tampoco era su lengua materna (japonesa, rusa o rumana). El alumnado del resto de grupos (castellano y valenciano) dominaba perfectamente el idioma de la asignatura. Por tanto, en total de estudiantes que siguen el curso de Derecho Procesal I en el grupo cuya docencia se imparte en inglés es de 51 . Por lo que respecta al grupo de valenciano (U), el grupo consta de 44 matriculados/as de los cuales 35 participaron en las pruebas de Kahoot. En cuanto al grupo de castellano (N) únicamente consta con 16 matriculados/as, de los cuales 9 participaron en la actividad a través de Kahoot.

En cuanto a los temas a abordar, se realizaron dos cuestionarios a través de Kahoot (que debieron ser uno en Kahoot y otro en Plickers). El primero versó sobre el Derecho de Defensa y el segundo sobre los Principios del Proceso. La elección de los temas reside en la importancia que tienen en los siguientes cursos de Derecho Procesal. El feedback inmediato de la aplicación Kahoot nos permite repasar aquellos conceptos que no han quedado claros por la complejidad de la asignatura en cursos iniciales.

\subsection{La utilidad de la gamificación en la docencia.}

Tal y como hemos puesto de manifiesto al inicio del presente trabajo, las nuevas tecnologías han irrumpido con fuerza en la docencia de todos los niveles educativos. Ello ha conllevado que las instituciones de educación superior se hayan visto obligadas a adaptar su infraestructura y sus políticas, pero también formar a profesores y estudiantes como parte de un plan tecnológico de adaptación a esta nueva realidad (Salinas, 2004). Desde el inicio, las llamadas TICs han servido como fuente para la innovación educativa a partir de la introducción de distintas herramientas tales como los juegos adaptados a la docencia, la creación de foros de debate virtuales, la utilización de las redes sociales 0 , de un modo general, la educación no presencial, tanto síncrona como asíncrona.

Una de las principales manifestaciones de esta revolución tecnológica es la aparición de herramientas de juego a través de medios telemáticos o gamificación, que es precisamente la herramienta que aquí nos ocupa. La gamificación ha sido tradicionalmente definida como el uso de los elementos y mecánicas del juego en contextos ajenos al juego, con la finalidad de estimular el interés, incentivar un cambio de comportamiento o transmitir un mensaje o contenido (Carreras, 2017, 109). Su empleo no se ha ceñido al ámbito estrictamente educativo, sino que en el ámbito empresarial ya se aplicaba incluso con anterioridad. Volviendo al ámbito educativo y partiendo de una concepción de la gamificación como técnica de innovación educativa podríamos decir que consiste en "aprender jugando".

Una de las claves del éxito de la introducción de estas técnicas de juego a través de medios tecnológicos en el ámbito educativo es su similitud con los videojuegos y otras plataformas a las que la juventud dedica un gran número de horas semanales. En este sentido, la introducción de herramientas de gamificación en la docencia, permitiría 
aprovechar las habilidades adquiridas a través de los videojuegos, al tiempo que se incrementa el interés y participación de los/las estudiantes (Carreras, 2017). Ello implica conectar ambos ámbitos, lúdico y educativo, de forma que el/la estudiante percibe que se trata de un juego, al tiempo que estimula su aprendizaje.

Por ello, la gamificación presenta incontables beneficios, tanto sobre el desarrollo de habilidades personales y profesionales, como en lo que respecta a la adquisición de conocimientos. Por un lado, podemos destacar la influencia sobre la motivación, el esfuerzo, la fidelización y la cooperación dentro del ámbito escolar (Andreu, 2020). Fundamentalmente dicha influencia positiva deviene del sentimiento de logro, reconocimiento y la propia competencia intrínseca al juego que estimula tales habilidades, así como otras potencialidades educativas (Sánchez, 2015). Por otro lado, la motivación que acompaña a la gamificación establece un vínculo entre el/la alumno/a y el contenido sobre el que se está trabajando, bien sea para absorber nuevos conocimientos, mejorar alguna habilidad o recompensar acciones concretas (Andreu, 2020).

En concreto, dentro de la gamificación de la docencia ha alcanzado una enorme popularidad en todos los niveles educativos y disciplinas la utilización de la herramienta Kahoot. Algunos de los factores que podrían justificar su crecimiento serían la facilidad para configurar por parte del/a profesor/a, así como la sencillez y el modo de juego para los estudiantes, que incrementa enormemente la atención y la participación, por la limitación de tiempo para contestar y la existencia tablas de puntuaciones en las que se visualizan los nombres de los/las participantes con mejor rendimiento. Además, únicamente se necesita tener un dispositivo con conexión a internet para poder participar.

Generalmente, la planificación de una sesión con Kahoot suele ser bien recibida por el estudiantado, pues supone la ruptura con las clases teóricas o prácticas habituales, lo que incrementa también su motivación. Además, la consolidación de conocimientos a través de la herramienta se refuerza si, tras la finalización del juego, se dedica un espacio de tiempo a comentar y debatir con los/las estudiantes el motivo para elegir una u otra respuesta y explicar los errores que se hayan podido cometer, a partir del feedback inmediato que ofrece la propia plataforma. Cabe destacar también que a través de Kahoot se produce una participación activa de todos/as los/las estudiantes en el proceso de aprendizaje, pues incluso los/las más tímidos/as obtienen un reconocimiento por el hecho de aparecer en el marcador final (Moya et al., 2016). Otra circunstancia que se ha revelado en experiencias con Kahoot es que incrementa significativamente la asistencia a clase (Sempere, 2018).

De todo lo expuesto, parece indudable que la gamificación aporta enormes beneficios a la docencia. No obstante, hay que señalar que, a pesar de todas las virtudes predicables de la gamificación a las que nos hemos referido, no puede negarse que ha habido una importante discusión en torno a su aplicación y eficacia a la educación superior, en concreto, a la educación universitaria. Como todo, ha tenido sus detractores/as y también sus seguidores/as, partiendo los primeros de la inutilidad del juego, frente a metodologías docentes tradicionales. Básicamente se ha criticado su capacidad para servir como método de aprendizaje en enseñanzas universitarias. Cierto es que, como cualquier herramienta docente, una inadecuada planificación puede dar lugar al fracaso de esta (Barros, 2016), lo que podrá deberse a la falta de atractivo para los/las alumnos, su baja participación o, sencillamente, su incapacidad para servir como herramienta de aprendizaje. 
Sin embargo, si partimos de una correcta planificación, las experiencias parecen demostrar precisamente su eficacia. En efecto, de acuerdo con estudio realizado por Andreu (2020) se desprende que la utilización de herramientas de gamificación sobre alumnos/as de enseñanzas universitarias arroja unos resultados positivos sobre la motivación del estudiantado. Especialmente, se refiere el autor, al impacto positivo sobre la motivación cuando se establece una puntuación, se otorgan insignias o aparecen tablas de clasificación de los/las estudiantes con mejor rendimiento en el juego (Andreu, 2020,90 ), a las cuales nos hemos referido supra. Este impacto es de vital importancia, pues la motivación es un factor determinante en el proceso de aprendizaje, ya que logra que los/las estudiantes participen, se involucren, presten atención y, en definitiva, desarrollen unos conocimientos que duren a un plazo mayor que los que se logran con una mera recepción pasiva de información (Vérez, 2016).

A todo ello debe sumarse la enorme utilidad de la información que el/la docente recoge de los informes que elabora automáticamente la plataforma Kahoot, no solamente en lo relativo a la participación y resultados individuales de cada alumno/a, sino respecto de los conceptos que menos asimilados tienen los/las estudiantes (esto es, las preguntas en las que mayores errores se registran), así como aquellos que están más consolidados e incluso si la redacción de alguna pregunta no es clara o adecuada para los objetivos del juego por ofrecer unos resultados que no corresponden con la realidad (Sempere, 2018).

Sin ánimo de adelantar las conclusiones del presente trabajo, sí que cabe señalar que los resultados corroboran lo expuesto, demostrando que la utilización de la herramienta Kahoot fomenta la participación del alumnado, incrementa su motivación y refuerza el aprendizaje, facilitando la asimilación y retención de los conceptos sobre los que se ha trabajado.

Asimismo, aun cuando será posteriormente cuando nos adentremos en las valoraciones por parte de los/las estudiantes del proyecto que aquí se presenta, sí que podemos ya anticipar que, en general, se han mostrado muy satisfechos con la herramienta Kahoot. Como posteriormente se expondrá en profundidad, los/las estudiantes han destacado fundamentalmente la utilidad de la herramienta para repasar y consolidar conceptos, máxime tras la discusión posterior en torno a los contenidos trabajados. Debe además destacarse que dicha valoración se ha obtenido a pesar de que, por las circunstancias concurrentes durante el mes de abril, se haya tenido que realizar la actividad de forma no presencial, todo lo cual se explica seguidamente. Ello implica que la percepción positiva sobre la utilidad de Kahoot es similar tanto en los/las docentes como en el estudiantado.

Por todo ello, podemos afirmar que, efectivamente, la gamificación resulta de gran utilidad para las enseñanzas universitarias de Grado y que, efectivamente, contribuye a la adquisición de conocimientos y el desarrollo de diversas habilidades tanto profesionales como personales, de conformidad con todo lo anteriormente expuesto.

\subsection{Covid-19 y docencia online}

La declaración del estado de alarma supuso la reestructuración de la docencia y la adaptación a la modalidad telemática en cuestión de días. La situación de excepcionalidad vivida por la pandemia ha impuesto el teletrabajo como única alternativa.

Así, en un intento por conservar actividades que iban a desarrollarse durante la docencia presencial junto a la intención de motivar al estudiantado y mantenerlo activo en el aprendizaje, pese a la distancia, la herramienta Kahoot deviene un instrumento de máxima utilidad.

Normalmente el Kahoot es una actividad de clase presencial, pero, dada su versatilidad, la propia aplicación permite dos sistemas "Enseñar" o "Asignar". El primero es más próximo a la experiencia en el aula. A través de Blackboard Collaborate la 
docente comparte la aplicación con el alumnado de forma que en la pantalla verán las preguntas y el contenido de las respuestas. Cada estudiante tendrá en su dispositivo móvil o en una segunda ventana en el ordenador la correspondencia de las respuestas en el formato de símbolos (triángulo rojo, rombo azul, esfera amarilla y cuadrado verde).

Se trata de jugar en vivo simulando la inmediación de la clase presencial, dado que se permite la interacción en directo. El/la profesor/a es quien activa y da paso a las preguntas, es posible la comunicación simultánea, así como inmediatamente después de su finalización para resolver dudas o realizar cualquier comentario.

En el segundo modelo se habilita el cuestionario para la realización individual en un período de tiempo concreto. Podría decirse que se establece un dies a quo y un dies ad quem para que los estudiantes completen el Kahoot. En diferido, viendo las preguntas y respuestas en sus dispositivos, se ajusta a las circunstancias personales del estudiante y se evitan los posibles problemas técnicos o de red sobrevenidos que pueden darse en el sistema anterior.

No obstante, en ambos supuestos la aplicación ordena y evalúa las respuestas, obteniendo el mismo resultado y permitiendo a el/la docente elegir el sistema que mejor le convenga y adapte a sus necesidades docentes.

Con todo, es necesario remarcar el carácter intuitivo y el uso gratuito de la plataforma, dos características a destacar en un contexto de aprendizaje apresurado. Además, es una herramienta cuya funcionalidad se hace depender de un dispositivo con conexión a internet, pudiendo crearse en cualquier momento y lugar. Es decir, es una herramienta que debe combinarse con unas mínimas habilidades y técnicas informáticas y de uso de las TICs en la enseñanza superior, pudiendo obtener un resultado muy satisfactorio.

Asimismo, cabe tener en cuenta que el grupo destinatario pertenece a la denominada generación de nativos/as digitales. Esta cualidad no solo facilita el desarrollo de la herramienta en términos de familiarización innata con la tecnología, sino que agiliza su implementación y la explicación correspondiente en un momento en el que hay que adaptarse a la docencia virtual, en plazos muy cortos, para garantizar la correcta finalización del curso académico.

En definitiva, la transición hacia una docencia semipresencial proyecta el uso de la tecnología en la docencia no solo como posible, sino más bien como necesario. Plataformas como Kahoot democratizan su uso.

\subsection{Objetivos específicos de la experiencia}

La realización de cuestionarios de la herramienta Kahoot en grupos de Derecho en los que se imparte la docencia de la asignatura de Derecho Procesal I (Introducción), que sienta las bases de la organización, estructura y tipología de procesos, en los tres idiomas oficiales de la Universitat de València (castellano, valenciano e inglés), pretende valorar los siguientes extremos:

- El idioma como factor determinante del aprendizaje, esto es, si ese factor de dificultad añadido se refleja en los resultados de las evaluaciones.

- La utilidad del Kahoot como metodología de aprendizaje más profundo o afianzado de determinados contenidos, especialmente en momentos clave como el actual, donde la crisis sanitaria ha obligado a imponer la docencia online como modalidad de enseñanza-aprendizaje. Ello tomando en consideración la dificultad de estudiar en idiomas distintos a la lengua materna. Con ello se podría analizar la viabilidad de estudiar Derecho Procesal en otros idiomas. 
- El análisis de la percepción de la utilidad del Kahoot en la adquisición de conceptos, su afianzamiento y en el desarrollo de otro tipo de competencias como el uso eficiente de las TICs.

\section{RESULTADOS}

Tomando en consideración los objetivos marcados, se exponen los resultados obtenidos, para cuya evaluación cabe tener en cuenta la heterogeneidad cuantitativa de la muestra.

\subsection{Primera temática: el derecho de defensa}

El primer cuestionario Kahoot versaba sobre el Derecho de Defensa y los principios procesales que de él se derivan. Como se observa en el gráfico siguiente, el promedio de aciertos de los tres grupos de docencia se sitúa en torno al $60 \%$. En particular, $61 \%$ en inglés, $58 \%$ en castellano y $57 \%$ en valenciano. Valores, todos ellos, elevados que hacen intuir el alto rendimiento en la materia de Derecho Procesal.

El balance de aciertos/errores, contenido en la gráfica, refleja el estudio previo de la materia por el alumnado. Un estudio que, además, se vio reforzado con la puesta en común de las respuestas una vez finalizada la experiencia, explicando aquellos conceptos dudosos. Si bien es cierto que solo hubo una o dos preguntas un poco más conflictivas, con un mayor número de errores, no lo es menos que la ventaja adicional que presenta el Kahoot es, precisamente, el feedback inmediato, que permite corregir la situación y aclarar tales aspectos. En ese intercambio de ideas entre docente y alumno/a se plantean dudas, se explican respuestas y se fomenta un aprendizaje dinámico.

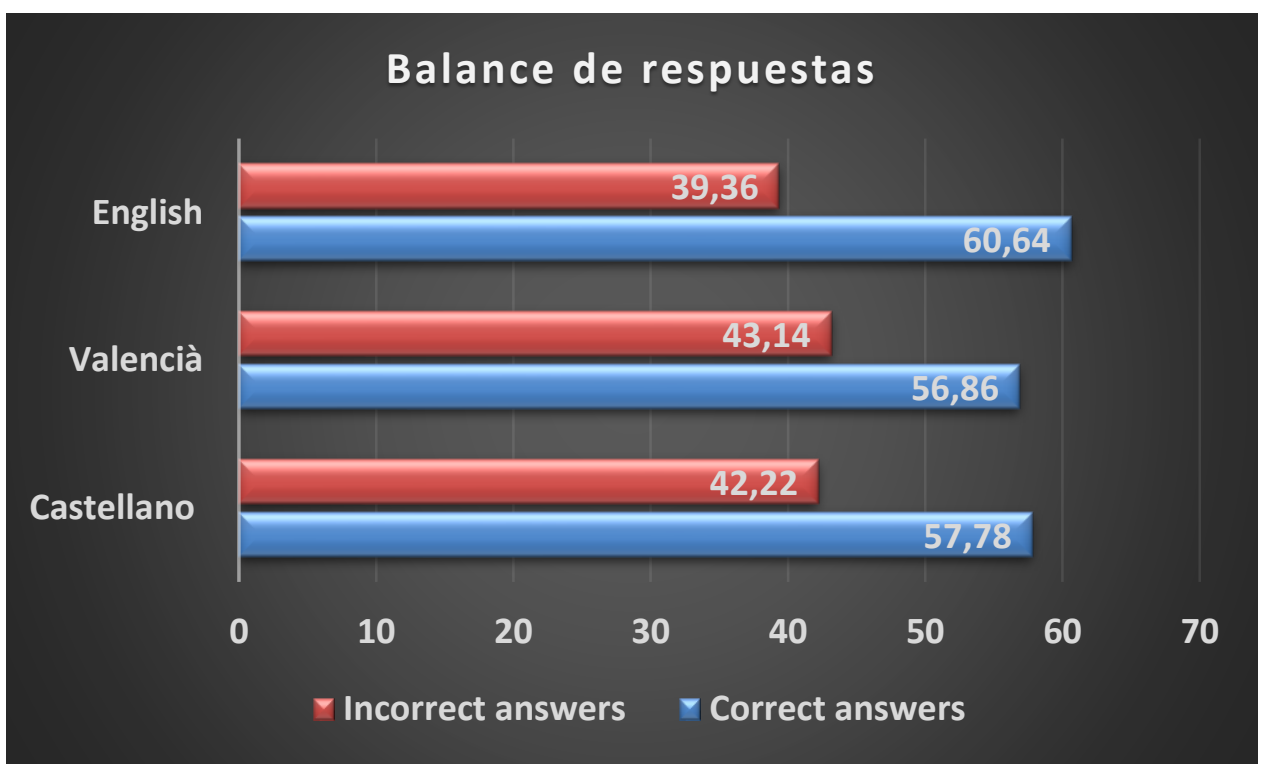

Figura 1. Balance de respuestas correctas/incorrectas de los cuestionarios.

Por otro lado, se analizan las puntuaciones obtenidas por el alumnado, reflejadas en el siguiente gráfico. Cabe justificar la obtención de una puntuación superior a 10.000 con una de las múltiples posibilidades que ofrece el Kahoot, que los/las participantes puedan recibir puntos de bonificación por las respuestas correctas consecutivas. Esto es, pese a que cada pregunta, de un total de diez, se contabilizaba con un valor máximo de 1.000 puntos, el hecho de conseguir una racha de respuestas correctas, se premia con hasta 500 puntos extra, lo que se conoce como "bonus points". De ahí que encontremos que la mejor valoración sea de 12.250 puntos, correspondiente a un/a estudiante del grupo de docencia en inglés. En total, hasta diez estudiantes 
pertenecientes a este grupo superaron la barrera de los 10.000, mientras que en el grupo de valenciano solamente lo hicieron dos estudiantes.

Si se atiende a las puntuaciones individuales obtenidas del gráfico, el grupo cuya docencia se imparte en inglés sigue encabezando la evaluación positiva a una distancia apreciable, especialmente respecto del grupo en castellano (12.250 frente a 7.904). Al comparar los valores individuales, la mejor puntuación en castellano se equipararía a las obtenidas en el segundo cuarto de la tabla del grupo en inglés y solo un estudiante de valenciano entraría hipotéticamente a ocupar un quinto puesto. Salvo en el grupo de valenciano que sí hay una puntuación que destaca, en el resto hay un reparto equilibrado entre puntuaciones muy buenas, buenas y regulares.

Podría pensarse que el tamaño de cada muestra, al existir una importante horquilla $-47,35$ y 9 estudiantes- influye en los resultados. Nada más lejos de la realidad, pues la gran ventaja de este método es la mayor objetivación de los resultados, al otorgar unos valores concretos en función de varios indicadores, tales como el acierto o fallo de la respuesta, la rapidez en responder e incluso la precisión por contestar correctamente varias preguntas seguidas -premiándose con una bonificación-. Por el contrario, el perfil del alumnado, esto es, edad, formación previa, experiencia laboral o incluso que se trate de una segunda matrícula en la asignatura, podría ser un factor relevante.

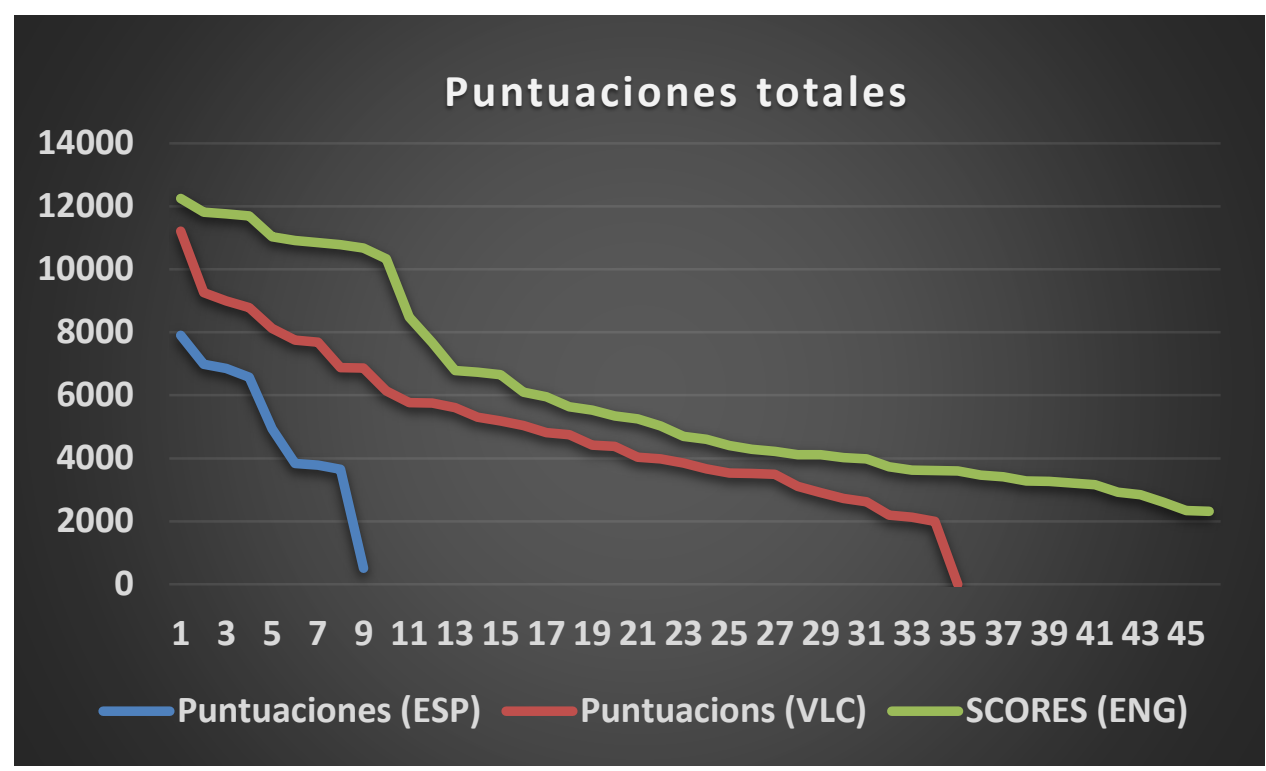

Figura 2. Evoluciones de las puntuaciones obtenidas por el alumnado.

En consecuencia, los datos extraídos demuestran que el grupo de docencia en inglés mantiene una elevada progresión de aciertos, superior a las de los grupos de docencia en castellano y en valenciano. Ello confirmaría un mayor afianzamiento de conocimientos y una mayor seguridad en el correcto aprendizaje de las bases y fundamentos del Derecho Procesal.

\subsection{Segunda temática: los principios del proceso}

La temática del segundo Kahoot se amplía a los principios del proceso, donde se invierte la ratio de aciertos de cada grupo con respecto al caso anterior, aunque sigue por encima del $60 \%$. Así, el grupo de inglés obtiene el porcentaje más bajo, si bien con una 
distancia muy reducida y siendo, en todo caso, superiores a los del primer Kahoot con un $66 \%$ en castellano, $64 \%$ en valenciano y un $60 \%$ en inglés.

El estudio de la asignatura en un idioma diferente a la lengua materna o vehicular requiere de un mayor esfuerzo, tanto para el claustro docente como para el alumnado, especialmente dada la complejidad derivada de conceptos básicos, instituciones y expresiones propiamente nacionales y carentes de una traducción o equivalente en ordenamientos anglosajones, alejándose las traducciones en algunos casos del término original, especialmente partiendo de una normativa elaborada en español.

No obstante, ambas experiencias demuestran una ratio aciertos/errores equilibrada y con buenos resultados, donde el idioma de impartición de la docencia no genera ningún tipo de desventaja para la adquisición de conocimientos en materia de Derecho Procesal, lo que quiebra cualquier crítica realizada al plurilingüismo y al Plan de Estudios de la carrera de Derecho en inglés. Entendemos que la desconfianza de enseñar en inglés podría derivar de la utilidad de cursar Derecho en un idioma distinto al de la propia litigación o la idiosincrasia de nuestro ordenamiento jurídico, pero no por considerar que la formación no sería completa u óptima.

Obviamente existe mayor dificultad en el proceso de enseñanza-aprendizaje cuando no se imparte o estudia en la lengua materna del/de la docente o del alumnado. Podría haber una mayor dificultad si la metodología y materiales seguidos fuesen exactamente iguales, pero dado que se adaptan y modifican en función de la lengua (especialmente inglés versus castellano/valenciano), finalmente resulta en un aprendizaje similar. Así, el aprendizaje es más lento y requiere de un sobreesfuerzo (innegable) por parte del alumnado y del/de la docente, pero si ese esfuerzo extra se focaliza y dirige correctamente a través de una elección apropiada de actividades para reforzar conceptos y aplicarlos en distintos escenarios jurídicos, los resultados han acabado siendo muy similares.

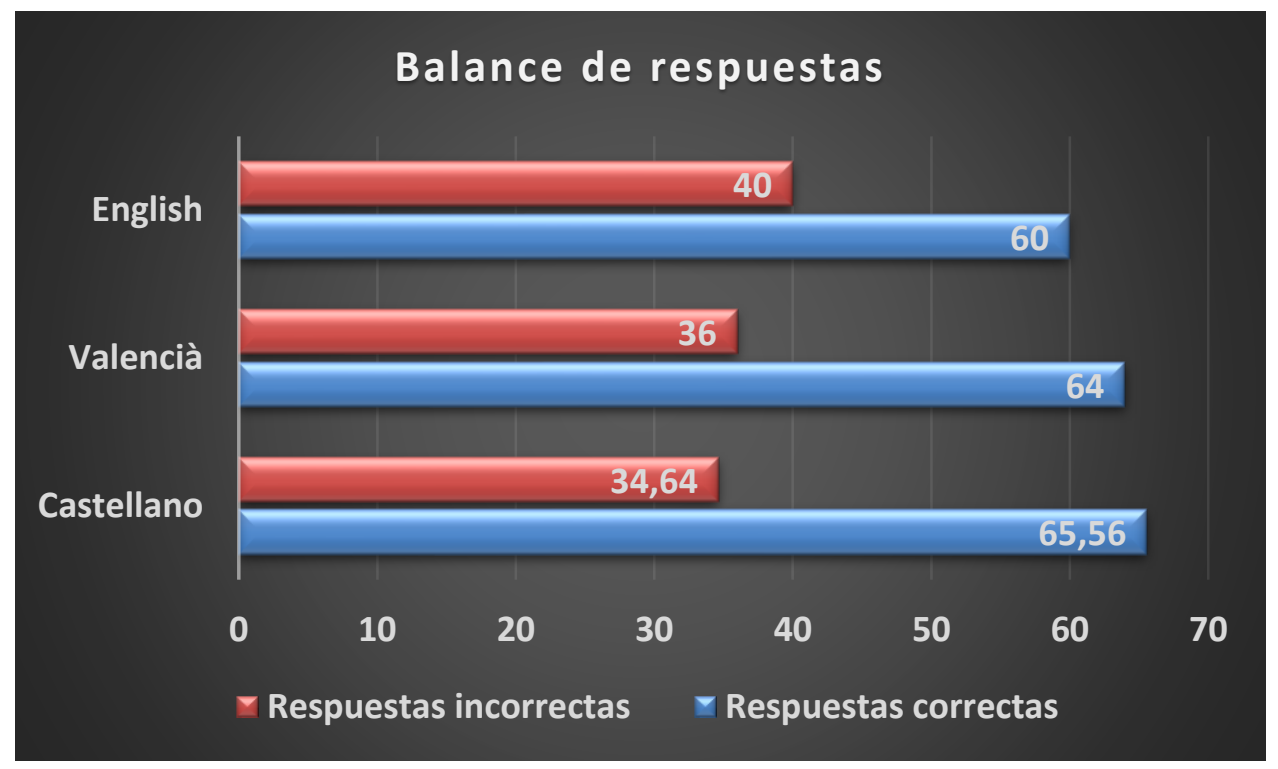

Figura 3. Balance de respuestas correctas/incorrectas de los cuestionarios.

En lo que concierne a las puntuaciones individuales, contenidas en el gráfico siguiente, el grupo de inglés sigue siendo superior a los otros dos, si bien la obtención de menos bonificaciones hace que la diferencia intergrupal no resulta tan significativa, siendo que, en este caso, las puntuaciones más bajas de los grupos de castellano y valenciano son superiores a las de inglés. 


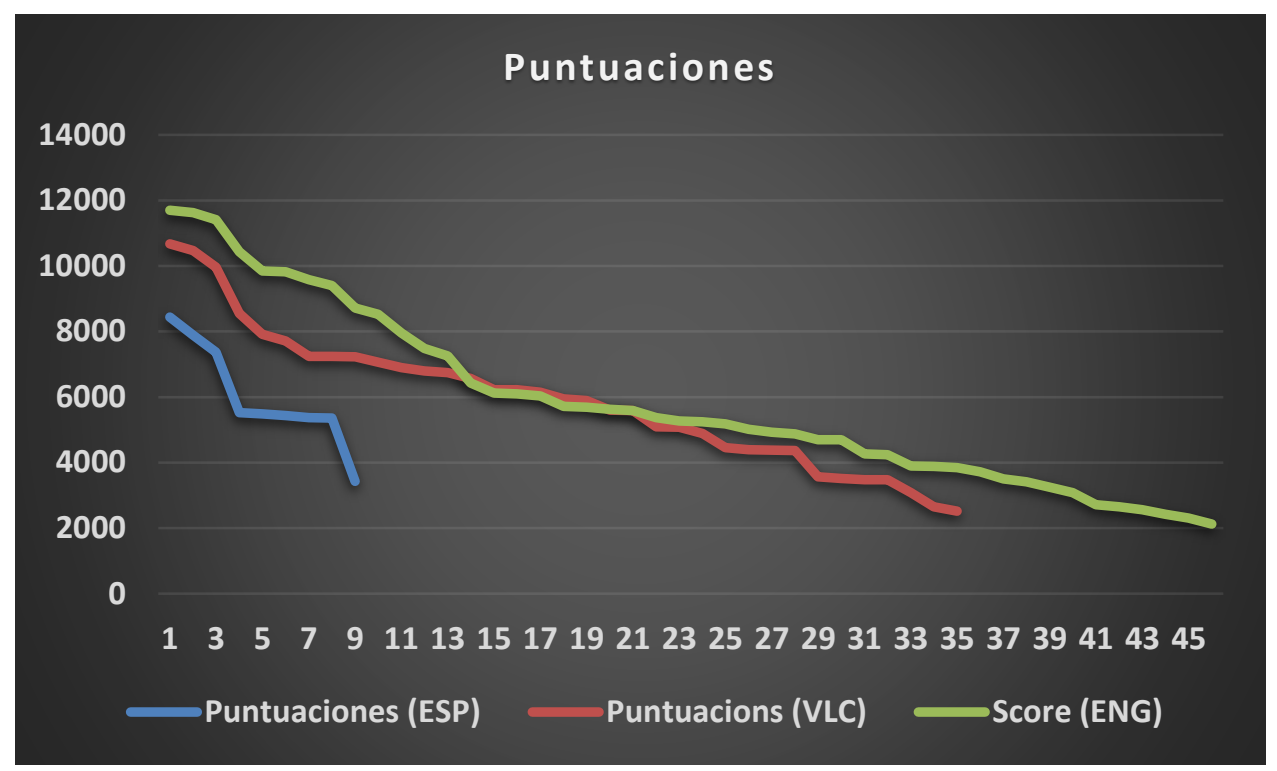

Figura 4. Evoluciones de las puntuaciones obtenidas por el alumnado.

Del diagnóstico de los resultados de los tres grupos en conjunto podemos confirmar que la ratio de aciertos/errores en los dos cuestionarios realizados es altamente positiva, superando el rango de aciertos el $60 \%$.

El factor clave a analizar a través de esta experiencia era la incidencia -positiva o negativa- sobre el rendimiento del alumnado del idioma de la docencia -castellano, valenciano e inglés-. La experiencia refuerza la idea de que el idioma no interfiere en el aprendizaje de Derecho Procesal ni merma la formación del alumnado, pues en esa línea el grupo de castellano tendría que haber sacado mejores resultados que el de inglés, y no al revés. La clave reside, más bien, en el perfil del alumnado, de su interés en la materia y de su participación en las distintas tareas y actividades planificadas.

Por último, no puede desconocerse que el empleo del Kahoot permite la adquisición de conocimientos en otras áreas que no son estrictamente la materia de Derecho objeto de estudio. El estudiantado mejora en el manejo de las TICs y se expone a la necesidad de aprender a adaptarse a nuevos contextos y escenarios, una cualidad esencial para su formación laboral. Dado que ha quedado resuelto que el idioma no supone un obstáculo para el aprendizaje, cabe subrayar que conocerlo sí reporta consecuencias beneficiosas. Entre ellas, la aproximación a una cultura diferente, el desarrollo de la escucha activa o la potenciación de la empatía y la comprensión por entender y hacerse entender.

\subsection{Satisfacción del alumnado con la experiencia}

El último objetivo de esta experiencia consiste en el análisis de la percepción de la utilidad del Kahoot en la adquisición de conceptos, su afianzamiento como método de enseñanza-aprendizaje y su eficacia en el desarrollo de otro tipo de competencias. Entendemos que el criterio y opinión del alumnado al respecto deviene fundamental para comprender mejor los resultados derivados de la práctica de las herramientas de gamificación como el Kahoot, especialmente en el estudio de la materia de Derecho Procesal. A tal fin se previeron dos mecanismos de evaluación de la satisfacción del alumnado con la experiencia, con el ánimo de obtener la máxima información posible. 
Por un lado, la propia herramienta Kahoot ofrece un feedback sobre la opinión del alumnado respecto de un doble aspecto. Primero, establece una ratio de 1 a 5 , siendo 1 el grado máximo de insatisfacción con la experiencia y 5 el máximo de satisfacción. Segundo, permite averiguar cómo se sienten los/las estudiantes con las puntuaciones obtenidas por sus respuestas a las distintas preguntas. Pregunta que nos parece muy interesante y que, en cierto modo, refleja no solo los puntos obtenidos, sino también la correspondencia entre tales puntuaciones y el nivel de estudio previo.

Por otro lado, quisimos que el alumnado tuviera la oportunidad de explicar con libertad, a través de un cuestionario de respuesta abierta planificado en la plataforma virtual del curso (Moodle), los puntos positivos y negativos de la experiencia, de su eficacia como mecanismo para controlar el nivel de aprendizaje y su importancia como método de enseñanza-aprendizaje.

El feedback obtenido por parte del alumnado es altamente positivo. Si bien comienzan a acostumbrarse al uso e incorporación de las nuevas tecnologías como herramientas de aprendizaje, aún siguen sorprendiéndose del uso docente de los teléfonos móviles, así como de su practicidad.

De esta forma, en primer lugar, cuando analizamos las valoraciones en la ratio de 1 a 5, observamos que en los tres grupos objeto de la experiencia se han obtenido puntuaciones superiores al 4 . Ello demuestra que es una actividad útil y recomendable para su aplicación en otros grupos de docencia, con independencia del idioma en que se imparta y de los puntos obtenidos por la realización del cuestionario. En efecto, estudiantes con malas calificaciones en el Kahoot han valorado positivamente la aplicabilidad de la herramienta para centrar y planificar su estudio, así como la dinámica de la clase y el aprendizaje resultante.

El gráfico siguiente refleja las puntuaciones concretas. Así, la media para el grupo de castellano fue la mayor de los tres, siendo de 4,8; seguida de la media del grupo de valenciano, de 4,6 y, finalmente, del grupo de inglés de 4,3.

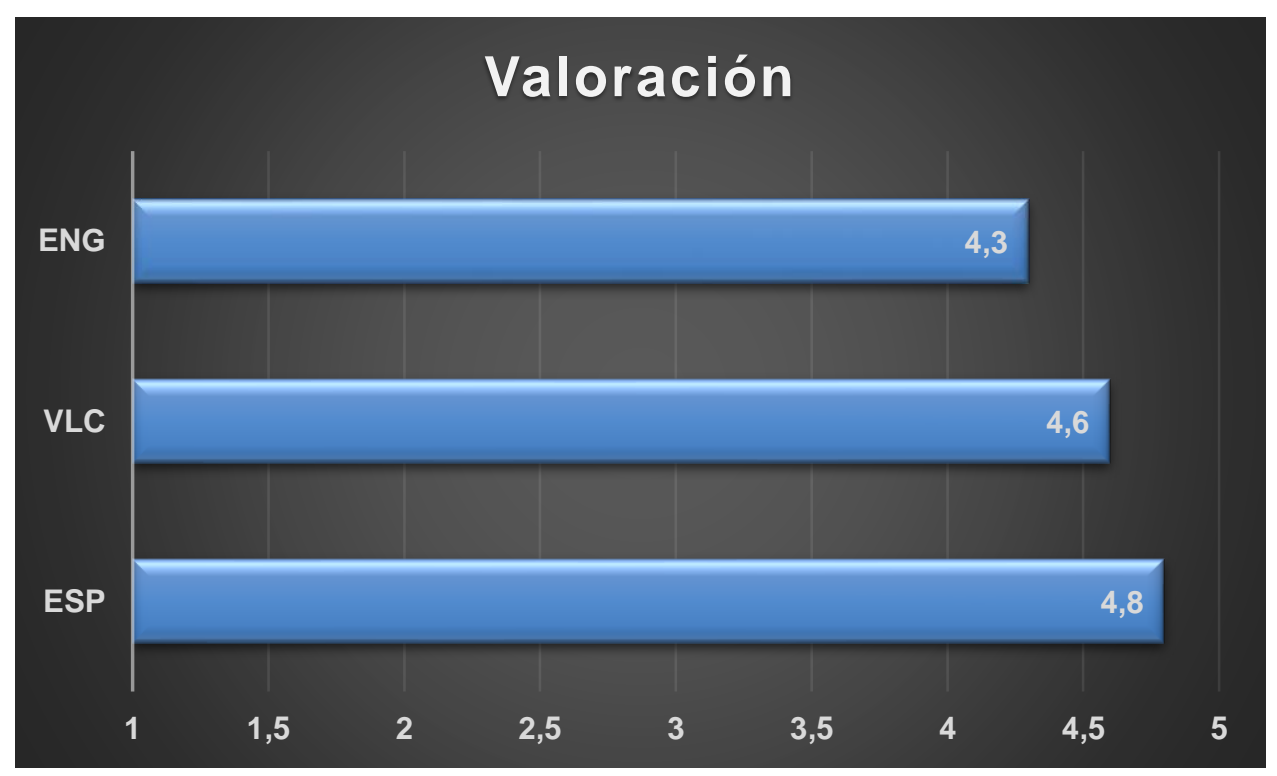

Figura 5. Valoración del alumnado de la satisfacción de la experiencia en el aprendizaje.

Valoraciones que debemos analizar conjuntamente con las opiniones vertidas por los/las estudiantes a través del cuestionario abierto sobre los pros y contras de esta herramienta y de los cuestionarios realizados.

Por un lado, consideran que la experiencia con el Kahoot les resulta todavía novedosa, pues aun cuando la gamificación en la enseñanza superior gana adeptos, sigue siendo utilizada en pocas materias. Estamos ante una herramienta, en opinión del 
alumnado, "entretenida", destacándose el aspecto visual de las respuestas y reflejando, todo ello, el dinamismo del propio cuestionario, que constituye un elemento motivacional. Dinamismo que genera un ambiente "divertido" y de "competitividad sana" entre compañeros/as, quienes buscan obtener la máxima puntuación, pero sin menospreciar a aquellos/as que no obtienen puntuaciones elevadas, pues el feedback de cada pregunta refleja las primeras posiciones y aquel participante que ha escalado más puestos o respondido rápidamente a la pregunta concreta. De esta forma, no se valora solo a quienes están en el pódium, sino también a aquellos/as participantes cuya rapidez en la respuesta merece ser reconocida. No obstante, La diversión derivada de esa competitividad generada entre el alumnado puede tornarse en perversión si no se conduce adecuadamente, impidiendo que únicamente importe la rapidez y la posición en el pódium, pero no el contenido y el porqué de la respuesta.

Ahora bien, el adjetivo que más resuena entre las respuestas de la encuesta de valoración, es "útil". Así es, prácticamente la totalidad de los/las participantes han resaltado la utilidad y practicidad del Kahoot como mecanismo para la consolidación de los conceptos enseñados durante las clases teórico-prácticas. El Kahoot resulta positivo no solo para incentivar al estudio de la asignatura durante el semestre, evitando el estudio de "última hora" para el examen, para aclarar conceptos, dudas o cuestiones del temario y comprobar el nivel de comprensión de la materia estudiada. Pero, sobre todo, deviene esencial para reorganizar el estudio, centrándose en aquellas lecciones cuyas preguntas han fallado o han tenido mayores dificultades. Es una buena forma de aprendizaje en todos los sentidos, pues aun cuando hayan errado en la respuesta, el feedback inmediato ofrece la respuesta correcta, lo que permite que el/la estudiante se percate de su error y aprenda del mismo.

Por otro lado, aunque estamos ante una herramienta tecnológica muy sencilla, no queda exenta de problemas técnicos. En este sentido, algunos/as estudiantes indican el bloqueo de alguna pregunta o respuesta, lo que les deja con menor margen y tiempo de respuesta. Otros/as, en cambio, señalan la dificultad de trabajar bajo presión, pues deben responder en un límite tan corto de tiempo (recuérdese que les dejamos 30-40 segundos para responder cada pregunta), y no consideran que se les deja margen suficiente para razonar debidamente las respuestas, lo que supone que en ocasiones fallen por no haber leído correctamente las preguntas. Aun así, valoran positivamente la experiencia por el aprendizaje.

Finalmente, y como indicamos supra, la propia herramienta permite conocer la satisfacción de los/las estudiantes, pero ahora en relación con sus propios resultados del Kahoot, aquellos obtenidos tras responder al cuestionario. Para ello, establece tres opciones: positivo, neutral y negativo. Opiniones, o feelings como se describe en la propia herramienta Kahoot, que denotan su interés y valoración personal.

A la vista del gráfico, se observa que de igual forma que se obtuvieron medias superiores al 4 en el análisis de la ratio, también encontramos un alto grado de satisfacción con el $78 \%$, seguido del $19 \%$ que tienen sentimientos calificados como "neutrales" y solo un 3\% ha mostrado su inconformidad y desaprobación del uso de la herramienta Kahoot. Obviamente entendemos que este 3\% coincide con las opiniones negativas manifestadas por algunos/as estudiantes relativas a la falta de concordancia entre el esfuerzo realizado durante la asignatura y el estudio previo con la puntuación o marcaje objetivo del cuestionario. Otros/as expresan también su desagrado por el estrés generado al limitarse el tiempo de cada pregunta a 30 ó 40 segundos, lo que les deja un pequeño margen para responder. Opiniones que son excepcionales frente a una mayoría que confirma la utilidad y eficacia del Kahoot como herramienta docente. 


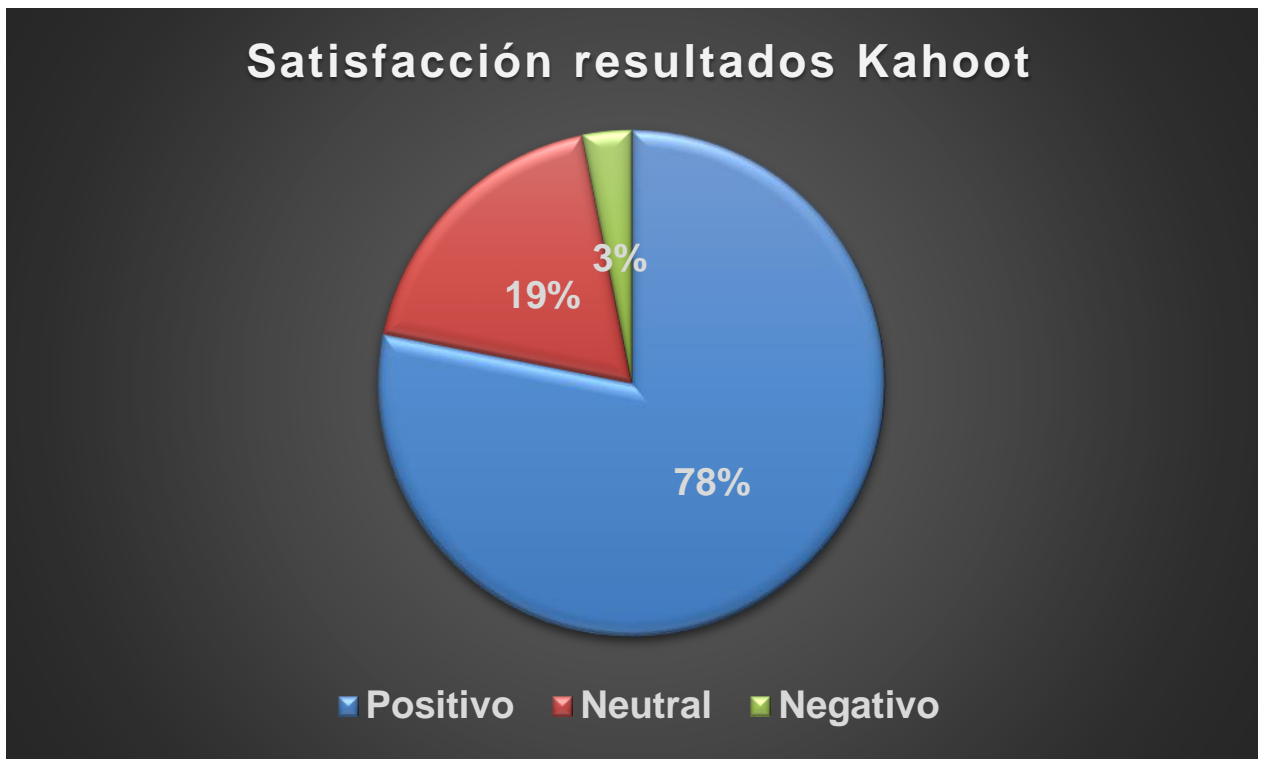

Figura 6. Feelings del alumnado respecto de sus resultados obtenidos en el Kahoot.

En suma, la experiencia arroja valoraciones muy positivas, redundando en una mejora en la dinámica del curso y de la posibilidad que ofrece -a través del feedback de resultados del cuestionario- de (re)planificar mejor el estudio para afrontar el examen de la asignatura y que, en opinión del alumnado, es una manera dinámica y entretenida de aprender, entender y reforzar los contenidos de la materia de Derecho Procesal a través de la "competitividad sana" entre ellos/as.

\section{CONCLUSIONES}

Estamos claramente ante una metodología que difiere de la clase magistral y de los debates tradicionales, donde normalmente es el/la docente quien ofrece el feedback al alumnado (Carreras, 2017), siendo más complicada la participación libre y continuada de éste. El uso de estas herramientas plantea ciertos retos (Carreras, 2017). Si bien es cierto que las técnicas docentes están en continua transformación, no lo es menos que debemos ser cautelosos con su incorporación en tanto en cuanto podrían pervertir el aprendizaje y convertirse en una herramienta de distracción (Vérez, 2016). Por ello, debemos identificar cuáles, dentro de la dilatada variedad de opciones disponible, son las más recomendables para el uso docente y los resultados de aprendizaje previstos, pues de lo que se trata es de encontrar sinergias donde profesorado y alumnado desarrollen un aprendizaje continuo, basado, por supuesto, en la colaboración e innovación (Dalmases, 2017).

La experiencia docente con Kahoot en los distintos grupos de Derecho Procesal nos permite alcanzar las siguientes conclusiones, a saber:

\section{La herramienta Kahoot es un método de enseñanza-aprendizaje útil con incidencia positiva en el aprendizaje}

La herramienta Kahoot ha demostrado ser útil, dinámica y divertida, que utiliza una metodología empírica-analítica que fomenta la motivación del alumnado para el aprendizaje, con independencia del idioma en que se imparta. Se crea un ambiente de apariencia competitiva, pero en sentido positivo, donde el alumnado busca acertar las respuestas para ocupar los primeros puestos del ranking, pero al mismo tiempo consolidando conceptos e identificando los puntos débiles sobre los que deben profundizar y dedicar más tiempo de estudio, mejorando su rendimiento académico. 


\section{Kahoot fomenta la participación del alumnado}

El dinamismo generado en clase, aunado a la competitividad sana que se deriva de la metodología, constituyen un incentivo para la participación del alumnado y su vinculación e interacción con el grupo.

\section{El feedback inmediato permite la consolidación de conceptos y la organización correcta del estudio}

Tras cada pregunta la plataforma Kahoot ofrece un feedback inmediato, donde además el/la estudiante no solo ve sus propios resultados sino también los del grupo, siendo un incentivo para la mayor implicación del colectivo y de motivación para mejorar. Así es, aumenta su implicación, pero también su participación e interés en la materia, especialmente teniendo en cuenta que el/la estudiante tratará de conseguir la máxima puntuación.

El aspecto más destacable del feedback inmediato es la oportunidad de comprobar qué aspectos de la asignatura necesitan refuerzo y cuáles tienen muy claros, lo que permite elaborar un cronograma de estudio ajustado a la realidad y mejorar el rendimiento del estudio.

\section{Es necesario incrementar el lapso de tiempo de respuesta para eliminar la perversión derivada del escaso margen de reflexión.}

El riesgo de perversión derivado del mayor interés en la rapidez de respuesta en lugar de en la propia respuesta, esto es, en el aprendizaje y afianzamiento de los contenidos y conceptos, podría eliminarse si se concede más tiempo por pregunta, consiguiendo que la competitividad generada sea "sana" y "divertida" y centrada en la reflexión y en el aprendizaje efectivo de la materia.

\section{El idioma no interfiere en el aprendizaje de Derecho Procesal.}

La experiencia demuestra que, contrariamente a lo que podría pensarse, el idioma de impartición de la docencia no interfiere en el desarrollo y ritmo de aprendizaje de los/las estudiantes. Si bien es cierto que supone y requiere un mayor esfuerzo por parte del/la docente y del alumnado para comprender las bases y fundamentos, no lo es menos que ello no merma la motivación ni el compromiso del alumnado con el aprendizaje de la materia. Los resultados avalan que el idioma no es óbice para un correcto y eficiente aprendizaje del Derecho Procesal.

\section{El Kahoot es una herramienta versátil adaptable a cualquier contexto docente.}

La herramienta ofrece posibilidades que permiten su uso en la docencia virtual. El doble sistema de juego simultáneo o en diferido, su manejo intuitivo y la propia sencillez en su puesta en práctica son factores que favorecen su utilización en modalidades de docencia no presenciales.

\section{REFERENCIAS}

Andreu, J. M. P. (2020). Una revisión sistemática sobre gamificación, motivación y aprendizaje en universitarios. Teoría de la Educación. Revista Interuniversitaria, 32(1), 73-99. 
Barros Lorenzo, M. (2016) "La gamificación en el aula de lengua extranjera. In Sainz García, A. M. (Dir.), El Español como lengua extranjera en Portugal II: retos de la enseñanza de lenguas cercanas (pp. 14-25), Ministerio de Educación, Cultura y Deporte.

Bastante Granell, V. \& Moreno García, L. (2020). Plataforma digital «ludoteca jurídica»: una apuesta por la «gamificación» en Derecho. Innovación Educativa y Metodologías Docentes, 21, 25-44.

Calabrese, L. (2015). Internet como espacio de juego: del game a la gamificación. Lúdicamente.Juego, formación y escuela, 4 (7).

Carreras, C. (2017). Del homo ludens a la gamificación. Quaderns de Filosofia, 4 (1), 107-118.

Catalán Gil, S. (2019). Gamificación y motivación: nuevas herramientas para la captación de talento. UCJC Business and Society Review (formerly Known As Universia Business Review), 16(3), 146-179.

Dalmases Muntané, A. (2017) Uso de la gamificación en la enseñanza de ELE. ELE en Red.

Deterding, S., Sicart, M., Nacke, L., O-Hara, K. \& Dixon, D. (2011): Gamification: Using Game Design Elements in Non-Gaming Contexts. Libro de actas de CHI 2011 Workshop Gamification: Using Game Design Elements in Non-Game Contexts.

De Mingo-López, D. V. y Vidal-Meliá, L. (2019). Actividades Kahoot! en el aula y satisfacción del alumnado. 3C TIC. Cuadernos de desarrollo aplicados a las TIC, 8(1), 96-115.

González González, C. S. (2014) Estrategias para trabajar la creatividad en la Educación Superior: pensamiento de diseño, aprendizaje basado en juegos y en proyectos. Revista de Educación a Distancia (RED), 40.

Lee, J. J. \& Hammer, J. (2011). Gamification in Education: What, How, Why Bother? Academic Exchange Quarterly, 15(2).

Moya Fuentes, M. \& Soler García, C. (2018) 114. La gamificación mediante herramientas virtuales de respuesta de audiencia: la experiencia de Socrative y Kahoot. En: Roig-Vila, R. (Ed.) El compromiso académico y social a través de la investigación e innovación educativas en la Enseñanza Superior (pp. 11541163). Octaedro Universidad, España.

Moya Fuentes, M., Carrasco Andrino, M., Jiménez Pascual, A., Ramón Martín, A. Soler García, C. \& Vaello López, M. T. (2016) El aprendizaje basado en juegos: experiencias docentes en la aplicación de la plataforma virtual "Kahoot". XIV Jornadas de Redes de Investigación en Docencia Universitaria, 1241-1254.

Olmos Vila, R. (2018). Kahoot! una aplicación para la gamificación en el aula. En REDINE (Ed.), Conference Proceedings EDUNOVATIC 2017 (pp. 862-869), Adaya Press.

Salinas, J. (2004). Innovación docente y uso de las TIC en la enseñanza universitaria. Revista de Universidad y Sociedad del Conocimiento, 1,1-16.

Sánchez Peris, F.J. (2015) Gamificación. EKS, 16 (2), 13-15.

Sempere Ferré, F. (2018). Kahoot como herramienta de autoevaluación en la universidad. En IN-RED 2018. IV Congreso Nacional de Innovación Educativa y Docencia en Red. Editorial Universitat Politècnica de València. 250-255.

Smith, S., (2011). This Game Sucks: How to Improve the Gamification of Education. EDUCAUSE Review, 467(1), 58-59.

Vérez Osorio, I. M. (2016). La gamificación en el aprendizaje de los estudiantes universitarios. Rastros Rostros, 18 (33), 27-38. 